\title{
The Interactions of Ferroelectric Domain Walls and Crystallographic Defects in the $\mathrm{PbTiO}_{3}$ Films
}

\author{
Y. Liu ${ }^{1}$, Y. L. Tang ${ }^{1}$, Y. L. Zhu ${ }^{1}$, W. Y. Wang ${ }^{1}$, and X. L. Ma ${ }^{1,2}$ \\ 1. Shenyang National Laboratory for Materials Science, Institute of Metal Research, Chinese Academy \\ of Sciences, Wenhua Road 72, 110016 Shenyang, China \\ 2. School of Materials Science and Engineering, Lanzhou University of Technology, Langongping Road \\ 287, 730050 Lanzhou, China
}

Both domain walls and lattice defects are known to have great influences on the properties of a ferroelectric, the detailed interactions between them and the resultant novel phenomena are still less known. In the tetragonal ferroelectrics, previous experimental investigations indicate that misfit dislocations form at film deposition temperature when $\mathrm{PbTiO}_{3}(\mathrm{PTO})$ is cubic with a relatively larger lattice constant compared to $\mathrm{SrTiO}_{3}(\mathrm{STO})$, while these dislocations may further promote the nucleation of $a$-domain [1,2]. However, these studies were mainly concentrated on the interactions between $90^{\circ}$ domain walls and dislocations near the hetero-interface, indicating that a systematic observation on the interactions between dislocations and $90^{\circ}$ domain walls is required.

In addition, the effects of $180^{\circ}$ and charged domain walls which exhibit little strain were less studied previously due to the lack of instrumental resolution. Although some theoretical calculations about the interaction between dislocations and $90^{\circ} / 180^{\circ}$ domain walls have been conducted [3], they are not revealed so far in experiments. Particularly, the atomic details and the local strain interactions were unattainable in these methods.

With the recent development of aberration-corrected (scanning) transmission electron microscope (Cs(S)TEM), direct mapping of domain walls at the atomic scale becomes possible [4-7]. For instance, systematic investigations on $180^{\circ}$ domain wall and $90^{\circ}$ charged domain wall in tetragonal ferroelectrics reveal the different width and polarization characteristics at these domain walls compared to the uncharged one. Nevertheless, spatial coupling between defects and domain wall in PTO film are still not well understood.

The PTO films with the thickness of $40 \mathrm{~nm}$ and $80 \mathrm{~nm}$, respectively, were deposited on STO (001) substrate by pulsed laser deposition technique. The $\operatorname{KrF}(\lambda=248 \mathrm{~nm})$ excimer with a laser energy density of $2 \mathrm{Jcm}^{-2}$ was used. Prior to deposition, the substrate was heated to $750^{\circ} \mathrm{C}$ and maintained for 5 min to clean the substrate surface and the target was pre-sputtered for $30 \mathrm{~min}$ to make sure their surfaces clean. When depositing PTO films, the substrate temperature was maintained at $650^{\circ} \mathrm{C}$. An oxygen pressure of $20 \mathrm{~Pa}$ and a laser repetition rate of $5 \mathrm{~Hz}$ were used. After deposition, the film was annealed in an oxygen pressure of $5 \times 10^{4} \mathrm{~Pa}$ at $650^{\circ} \mathrm{C}$ for $10 \mathrm{~min}$, and then cooled down to room temperature with a cooling rate of about $5^{\circ} \mathrm{C} / \mathrm{min}$.

Basis on Cs-(S)TEM imaging, we extensively analyze domain patterns and unusual strain state caused by the complex interactions between lattice defects and ferroelectric domains in PTO films [8]. We find that: (1) a specific defect-domain wall interaction may give rise to a metastable domain configuration, such as unstable $a$ - and $c$-domain, charged $90^{\circ}$ domain walls as well as super-domain patterns. The relaxation of such metastable domain configurations is observed which may induce retention failure; (2) 
$90^{\circ}$ domain walls are usually accompanied with specific dislocations along the $90^{\circ}$ domain walls. In addition, unusual dislocation strain field which is along $90^{\circ}$ domain wall is revealed. Such a strain field deviates $45^{\circ}$ from that of the typical dislocation with an $a[0 \overline{1} 0]$ vector [9].

References:

[1] T Kiguchi et al., Sci. Technol. Adv. Mat. 12, (2011), 034413.

[2] D Su et al., Appl. Phys. Lett. 99, (2011), 102902.

[3] A Kontsos and CM Landis, Int. J. Solids Struct. 46, (2009), 1491.

[4] CL Jia et al., Nat. Mater. 7 (2008), P57.

[5] CT Nelson et al., Nano Lett. 11, (2011), P828.

[6] YL Tang et al., Sci. Rep. 4 (2014), 04115.

[7] YL Tang et al., Science 348 (2015), P547.

[8] Y Liu et al., Adv. Mater. Interfaces (2016), DOI: 10.1002/admi.201600342.

[9] The authors acknowledge funding from the National Natural Science Foundation of China (No. 51231007, 51571197, 51501194, 51671194 and 51521091), National Basic Research Program of China (2014CB921002), and the Key Research Program of Frontier Sciences CAS (QYZDJ-SSW-JSC010). YL Tang acknowledges the IMR SYNL-T.S. Kê Research Fellowship and the Youth Innovation Promotion Association CAS (No. 2016177).

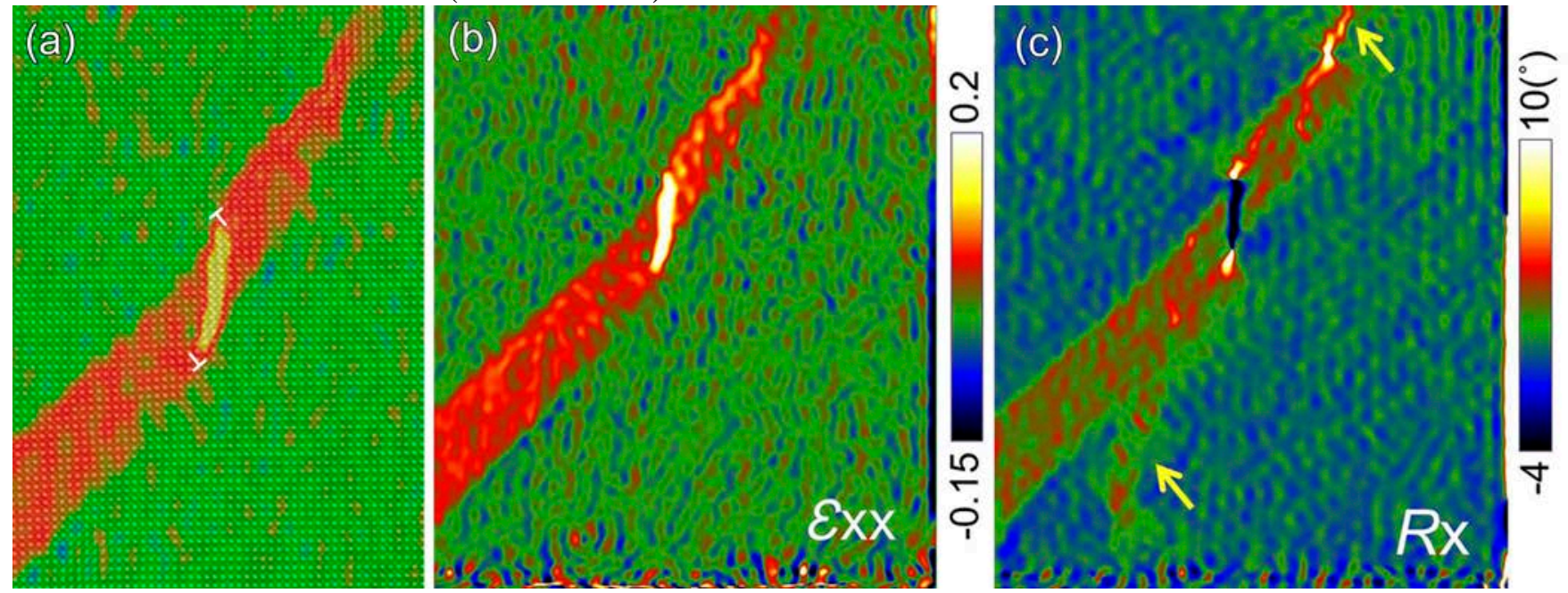

Figure 1. (a) A superimposition of HAADF-STEM images of $\mathrm{PbTiO}_{3}$ and corresponding in-plane lattice strain map, where a-domain is in red and $c$-domain is in green. The notation of " $\mathrm{T}$ ” denotes dislocations. This image implies that $a$-domain changes its extending direction when it comes across dislocations. (b) In-plane strain $\left(\varepsilon_{x x}\right)$ and (c) lattice rotation $\left(R_{x}\right)$ maps of (a) [8]. 